\title{
Interpreting the human genome sequence, using stochastic grammars
}

\author{
Richard Durbin \\ The Sanger Centre \\ Wellcome Trust Genome Campus \\ Hinxton \\ Cambridge CB10 1SA \\ UK \\ rdesanger.ac.uk
}

\begin{abstract}
The 3 billion base pair sequence of the human genome is now available, and attention is focusing on annotating it to extract biological meaning. I will discuss what we have obtained, and the methods that are being used to analyse biological sequences. In particular I will discuss approaches using stochastic grammars analogous to those used in computational linguistics, both for gene finding and protein family classification.
\end{abstract}

